\title{
Hamiltonian and self-adjoint control systems
}

\section{A. van der SCHAFT}

Dept. of Applied Mathematics, Twente University of Technology, P.O. Box 217, 7500 AE Enschede, The Netherlands

\section{P.E. CROUCH *}

Dept. of Electrical and Computer Engineering,

Arizona State University, Tempe, AZ 85287, USA

Received 22 May 1986

Revised 16 October 1986

Abstract: This paper outlines results recently obtained in the problem of determining when an input-output map has a Hamiltonian realization. The results are obtained in terms of variations of the system trajectories, as in the solution of the Inverse Problem in Classical Mechanics. The variational and adjoint systems are introduced for any given nonlinear system, and self-adjointness defined. Under appropriate conditions self-adjointness characterizes Hamiltonian systems. A further characterization is given directly in terms of variations in the input and output trajectories, proving an earlier conjecture by the first author.

Keywords: Hamiltonian system, Minimality, Lagrangian submanifolds, Symplectic.

\section{Introduction}

In this paper we present a brief review of some recently obtained results on the characterization of Hamiltonian control systems. A detailed treatment, including all the proofs, will be given elsewhere [6]. Consider a nonlinear control system (affine in the control $u$ ) on a $k$-dimensional manifold $M$,

$$
\begin{aligned}
& \dot{x}=g_{0}(x)+\sum_{j=1}^{m} u_{j} g_{j}(x), \\
& x(0)=x_{0} \in M, \quad u=\left(u_{1}, \ldots, u_{m}\right) \in R^{m}, \\
& y_{j}=H_{j}(x), \quad j=1, \ldots, p, \\
& y=\left(y_{1}, \ldots, y_{p}\right) \in R^{p} .
\end{aligned}
$$

(1.1) is called a Hamiltonian system if it is endo-

\footnotetext{
* Partially Supported by AFOSR \#85-0224A.
}

wed with the following additional structure. $M$ has to be a symplectic manifold with symplectic form $\omega$. For any function $H: M \rightarrow R$ the globally Hamiltonian vector field $X_{H}$ is defined by

$\omega\left(X_{H}, Z\right)=-\mathrm{d} H(Z)$, for any vector field $Z$.

By Darboux's theorem there exist local (canonical) coordinates $\left(q_{1}, \ldots, q_{n}, p_{1}, \ldots, p_{n}\right)$ for $M$ in which

$\omega=\sum_{i=1}^{n} \mathrm{~d} p_{i} \wedge \mathrm{d} q_{i}$

(so $k=2 n$ ). Then (1.2) amounts to the familiar expressions

$\dot{p}_{i}=-\frac{\partial H}{\partial q_{i}}, \quad \dot{q}_{i}=\frac{\partial H}{\partial p_{i}}, \quad i=1, \ldots, n$.

On the other hand if a vector field $g$ satisfies

$L_{g} \omega=0 \quad$ ( $L$ is Lie derivative),

then locally there will exist a function $H$ such that $g=X_{H}$, and $g$ is called a (locally) Hamiltonian vector field. Now assume that in (1.1) $g_{0}$ is a (locally) Hamiltonian vector field, i.e. $g_{0}=X_{H_{0}}$ for some locally defined function $H_{0}$ on $M$, and that $-g_{j}$ is equal to the globally Hamiltonian vector field $X_{H}, j=1, \ldots, p$, where $p=m$. Then (1.1) becomes the Hamiltonian system $[2,10,11]$

$$
\begin{aligned}
& \dot{x}=X_{H_{0}}(x)-\sum_{j=1}^{m} u_{j} X_{H_{j}}(x), \\
& x(0)=x_{0} \in(M, \omega), \quad u=\left(u_{1}, \ldots, u_{m}\right) \in R^{m}, \\
& y_{j}=H_{j}(x), \quad j=1, \ldots, m, \\
& y=\left(y_{1}, \ldots, y_{m}\right) \in R^{m} .
\end{aligned}
$$

If $H_{0}$ in (1.5) can be globally defined then (1.5) is called a globally Hamiltonian system. Hamiltonian systems are an appealing class of nonlinear systems, and are encountered in many physically oriented applications and also play a role in optimal control and filtering problems.

The central question of this paper is the following. Given a nonlinear system (1:1), when is it 
actually a Hamiltonian system (1.5), i.e., when does there exist a symplectic form $\omega$ on $M$ such that with respect to this $\omega$ we have

$L_{g_{0}} \omega=0$,

$\omega\left(g_{j}, Z\right)=-\mathrm{d} H_{j}(Z), \quad j=1, \ldots, m$,

$Z$ a vector field?

From a system-theoretic point of view this is a version of the Hamiltonian realization problem as initiated in [2,11]. As explained in [6,11] it can also be interpreted as a direct generalization of one of the fundamentals of mechanics, namely the Inverse Problem in Classical Mechanics [9]. For connections with a theory of quantization of control systems to quantum mechanical control systems we refer to [12]. In case (1.1) is a linear system

$\dot{x}=A x+B u, \quad y=C x$,

the answer to the above question is simple [3]: A minimal linear system is Hamiltonian if and only if the impulse response matrix $W(t)=C \mathrm{e}^{A t} B$ satisfies

$W(t)=-W^{\mathrm{T}}(-t), \quad t \geqslant 0$.

It was noted [11] that (1.7) can be replaced by the following appealing condition: A minimal linear system is Hamiltonian if and only if for every two input functions $u_{1}$ and $u_{2}$ with compact support, such that the corresponding output functions $y_{1}$ and $y_{2}$ also have compact support, we have

$\int_{-\infty}^{+\infty}\left[y_{2}^{\mathrm{T}}(t) u_{1}(t)-y_{1}^{\mathrm{T}}(t) u_{2}(t)\right] \mathrm{d} t=0$.

This led the first author to formulate a conjecture for the characterization of nonlinear Hamiltonian systems entailing a condition similar to (1.8) for the variational inputs and outputs, cf. [11]. In Section 3 we shall state a (slightly modified) version of this conjecture as Theorem 3.6. In fact, finding necessary and sufficient conditions for a nonlinear input-output map to be realizable by a Hamiltonian system, was solved by Jakubczyk [8], while already in Crouch \& Irving [5] conditions were found for a finite Volterra series to be realizable by a (nilpotent) Hamiltonian system. The connection between such an input-output approach and the variational approach taken in this paper is still open (see $[4,6])$. However en-route to Theorem 3.6 an intermediate result is stated, The- orem 2.3, which explicitly characterizes Hamiltonian systems by a direct generalization of the classical Helmholtz conditions for the Inverse Problem [9]. This requires the use of the variational and adjoint systems along every trajectory of the nonlinear system and the introduction of the notions of self-adjointness and of the prolongation and Hamiltonian extension of a nonlinear system. This is reviewed in Section 2.

The reader is urged first of all to briefly review our final comments and formal result, Theorem 3.8 , namely a minimal system is Hamiltonian if and only if its external behavior set of input-output pairs is a Lagrangian submanifold of the space of all input-output pairs, since this is the underlying reason for the validity of the following results.

In this paper we shall make two standing assumptions concerning the systems (1.1).

Assumption 1. The manifold $M$, the vector fields $g_{0}, g_{1}, \ldots, g_{m}$ and the functions $H_{1}, \ldots, H_{p}$ are (real-) analytic.

Assumption 2. The vector fields

$g_{0}+\sum_{j=1}^{m} u_{j} g_{j}$

for any constant $\left(u_{1}, \ldots, u_{m}\right) \in R^{m}$ are complete.

\section{The self-adjointness criterion}

Consider a nonlinear system (1.1). Take an arbitrary, but fixed, input function $u(t), t \in[0, T]$, such that the solution $x(t)$ of (1.1) remains within one coordinate neighbourhood of $M$. This also yields an output $y(t), t \in[0, T]$. Along this input-state-output trajectory $(u, x, y)$, the variational system is given by

$$
\begin{aligned}
& \dot{v}(t)= D g_{0}(x(t)) v(t) \\
&+\sum_{j=1}^{m} u_{j}(t) D g_{j}(x(t)) v(t) \\
&+\sum_{j=1}^{m} u_{j}^{v}(t) g_{j}(x(t)), \\
& y_{j}^{v}(t)= D H_{j}(x(t)) v(t), \\
& j=1, \ldots, p, \quad v(0)=0 \in R^{k},
\end{aligned}
$$


where $D$ denotes taking the Jacobian matrix. Furthermore

$u^{v}=\left(u_{1}^{v}, \ldots, u_{m}^{v}\right)$ and $y^{v}=\left(y_{1}^{v}, \ldots, y_{p}^{v}\right)$

denote the inputs and outputs of the variational system, and are called the variational inputs and outputs. This nomenclature is explained as follows. Let

$(u(t, \varepsilon), x(t, \varepsilon), y(t, \varepsilon)), \quad t \in[0, T]$,

be a one-parameter family of solutions of (1.1) with $u(t, 0)=u(t), x(t, 0)=x(t)$ and $y(t, 0)=$ $\nu(t), t \in[0, T]$ (this is called a variation of $(u, x, y)$ ). The following variational quantities then satisfy (2.1):

$$
\begin{gathered}
v(t)=\frac{\partial x(t, 0)}{\partial \varepsilon}=\delta x(t), \\
u^{v}(t)=\frac{\partial u(t, 0)}{\partial \varepsilon}=\delta u(t), \\
y^{\nu}(t)=\frac{\partial y(t, 0)}{\partial \varepsilon}=\partial y(t)
\end{gathered}
$$

Along this same trajectory

$$
(u(t), x(t), y(t)), \quad t \in[0, T],
$$

the adjoint system is defined as the dual linear time-varying system

$$
\begin{gathered}
-\dot{p}(t)=D g_{0}^{\mathrm{T}}(x(t)) p(t) \\
+\sum_{j=1}^{m} u_{j}(t) D g_{j}^{\mathrm{T}}(x(t)) p(t) \\
+\sum_{j=1}^{p} u_{j}^{a}(t) D H_{j}^{\mathrm{T}}(x(t)), \\
y_{j}^{a}(t)=g_{j}^{\mathrm{T}}(x(t)) p(t), \\
j=1, \ldots, m, \quad p(0)=0 \in R^{k},
\end{gathered}
$$

with inputs $u^{a}=\left(u_{1}^{a}, \ldots, u_{p}^{a}\right)$ and outputs $y^{a}=$ $\left(y_{1}^{a}, \ldots, y_{m}^{a}\right)$. For any input functions $u^{v}(t)$ and $u^{a}(t)$ it follows from (2.1) and (2.3) that

$$
\frac{\mathrm{d}}{\mathrm{d} t} p^{\mathrm{T}}(t) v(t)=\left(y^{a}(t)\right)^{\mathrm{T}} u^{\nu}(t)-\left(y^{v}(t)\right)^{\mathrm{T}} u^{a}(t)
$$

Moreover, if a system with inputs $u^{a}$ and outputs $y^{a}$ satisfies (2.4) for any $u^{v}$ and $y^{v}$ then it is equal to the adjoint system [16]. Hence the adjoint system is uniquely determined by the variational system. The variational and adjoint systems are only defined locally along a trajectory $(u(t), x(t)$, $y(t)), t \in[a, b]$, such that $x(t)$ remains within one coordinate neighbourhood. However, global (and coordinate-free) definitions can be given if we combine the original system together with all its variational or adjoint systems. Equations (1.1) together with (2.1) define the prolonged system, or prolongation, which has state space TM (local coordinates $(x, v)$ ), input space $T R^{m}$ (local coordinates $\left(u, u^{v}\right)$ ) and output space $T R^{p}$ (local coordinates $\left(y, y^{v}\right)$ ). Equations (1.1) together with (2.3) define the Hamiltonian extension, which has state space $T^{*} M$ (local coordinates $(x, p)$ ), input space $R^{m} \times R^{p}$ (local coordinates $\left(u, u^{a}\right)$ ) and output space $R^{p} \times R^{m}$ (local coordinates $\left(y, y^{a}\right)$ ).

Recall that in [13] a system (1.1) is said to be minimal if it is observable and orbit minimal. Since we assume analyticity we may alternatively describe orbit minimality via the Lie algebra $L$ generated by $g_{0}, g_{1}, \ldots, g_{m}$, namely we require that the system be accessible or $L(x)=T_{x} M$ for all $x \in M$. If $L_{0}$ denotes the ideal in $L$ generated by $g_{1}, \ldots, g_{m}$ then we say the system is strongly accessible if $L_{0}(x)=T_{x} M$ for all $x \in M$.

Because an observable and accessible Hamiltonian system is necessarily strongly accessible, we shall henceforth call a system minimal if it is observable and strongly accessible.

A remarkable fact concerning the prolongation and Hamiltonian extension is contained in the following result also proved in [6].

Theorem 2.1. A system (1.1) is minimal if and only if the prolongation is minimal, if and only if the Hamiltonian extension is minimal.

We shall only deal with piecewise constant, right-continuous input functions $u$. As a consequence of Assumption 2 the solution $x$ of (1.1) and corresponding output $y$ are defined on any interval of time for which $u$ is.

The input-output map of the variational system along the resulting trajectory $(u(t), x(t)$, $y(t)), t \geqslant 0$, is given by

$y^{v}(t)=\int_{0}^{t} W_{v}(t, \sigma, u) u^{v}(\sigma) \mathrm{d} \sigma, \quad t, \sigma \geqslant 0$,

where $W_{v}(t, \sigma, u)$ is the $p \times m$ matrix with $(i, j)$ th element

$D H_{i}(x(t)) \Phi^{u}(t, \sigma) g_{j}(x(\sigma))$ 
and $\Phi^{u}(t, \sigma)$ is the transition matrix for the timevarying linear system defined by the matrix

$D g_{0}(x(t))+\sum_{j=1}^{m} u_{j}(t) D g_{j}(x(t))$.

It is easily seen that $W_{v}(t, \sigma, u)$ exists for all $t, \sigma \geqslant 0$ and also can be defined in a coordinatefree way [6]. Similarly, the input-output map of the adjoint system is given by

$y^{a}(t)=\int_{0}^{t} W_{a}(t, \sigma, u) u^{a}(\sigma) \mathrm{d} \sigma, \quad t, \sigma \geqslant 0$,

and $W_{a}(t, \sigma, u)$ is determined by $W_{v}(t, \sigma, u)$ since [6]

$W_{a}(t, \sigma, u)=-W_{v}^{\mathrm{T}}(\sigma, t, u)$ for all $u$.

Definition 2.2. A variational system along an input $u$ is called self-adjoint if $p=m$ and

$W_{v}(t, \sigma, u)=W_{a}(t, \sigma, u)$

$\left(=-W_{v}^{\mathrm{T}}(\sigma, t, u)\right)$ for all $t, \sigma \geqslant 0$.

We can now state the main result of this section proved in [6].

Theorem 2.3. A minimal system (1.1) is Hamiltonian if and only if the variational systems along all piecewise constant inputs are self-adjoint.

Necessity in this result is straightforward. The proof of sufficiency is more involved but follows by applying Theorem 2.1 and Sussmann's uniqueness theorem on minimal realizations [13] to the prolongation and Hamiltonian extension.

\section{The variational criterion}

Consider again the nonlinear system (1.1), where we assume already that $p=m$. The set of all trajectories $(u(t), x(t), y(t)), t \geqslant 0$, will be denoted by $\Sigma_{\mathrm{i}}^{+}(0)\left(x_{0}\right)$. By omitting the state functions $x(t)$ we obtain the set of all possible input-output pairs $(u(t), y(t)), t \geqslant 0$, denoted by $\Sigma_{\mathrm{e}}^{+}(0)\left(x_{0}\right)$ (e from external behavior). Note that $\Sigma_{e}^{+}(0)\left(x_{0}\right)$ can be identified with the input-output map of (1.1). A variation of an element $(\bar{u}, \bar{x}, \bar{y})$ $\in \Sigma_{\mathrm{i}}^{+}(0)\left(x_{0}\right)$ will be a one-parameter family $(u(t, \varepsilon), x(t, \varepsilon), y(t, \varepsilon)) \in \Sigma_{\mathrm{i}}^{+}(0)\left(x_{0}\right)$,

such that $u(t, 0)=\bar{u}(t), t \geqslant 0$, and consequently $x(t, 0)=\bar{x}(t), \quad y(t, 0)=\bar{y}(t)$. Since we are only considering piecewise constant inputs the variations of $\bar{u}$ can be restricted to variations of the form

$u(t, \varepsilon)=\bar{u}(t)+\varepsilon \delta u(t)$

with $\delta u(t)$ an arbitrary piecewise constant rightcontinuous function.

Recalling the definition of $\delta u, \delta x, \delta y$ in (2.2) we shall often abuse notation and refer to $(\delta u, \delta x, \delta y)$ as a variation of $(\bar{u}, \bar{x}, \bar{y}) \in$ $\Sigma_{i}^{+}(0)\left(x_{0}\right)$, and $(\delta u, \delta y)$ as a variation of $(\bar{u}, \bar{y})$. The class of variations we work with is given in the following definition.

Definition 3.1. $(\delta u, \delta y)$ is called an admissible variation of compact support of $(\bar{u}, \bar{y}) \in \Sigma_{\mathrm{e}}^{+}(0)\left(x_{0}\right)$ if

(i) $\delta u(0)=0$, and supp $\delta u$ (support of $\delta u$ ) is compact.

(ii) supp $\delta y \subset \operatorname{supp} \delta u$.

(iii) Let supp $\delta u \subset(0, T)$, and let

$\left(\bar{u}^{\prime}, \bar{y}^{\prime}\right) \in \Sigma_{\mathbf{c}}^{+}(0)\left(x_{0}\right)$

be such that $\bar{u}^{\prime}(t)=\bar{u}(t)$ and hence $\bar{y}^{\prime}(t)=\bar{y}(t)$ for $t \in[0, T]$. Define a variation $u^{\prime}(t, \varepsilon)$ of $\vec{u}^{\prime}$ by setting

$u^{\prime}(t, \varepsilon)=\bar{u}^{\prime}(t)+\varepsilon \delta u(t)$.

This yields a variation $\left(u^{\prime}(t, \varepsilon), y^{\prime}(t, \varepsilon)\right)$ of $\left(\bar{u}^{\prime}, \bar{y}^{\prime}\right)$. We require that the resulting (infinitesimal) variation $\left(\delta u^{\prime}, \delta y^{\prime}\right)$ of $\left(\bar{u}, \bar{y}^{\prime}\right)$ also satisfies (ii), i.e.

$\operatorname{supp} \delta y^{\prime} \subset \operatorname{supp} \delta u^{\prime}=\operatorname{supp} \delta u$.

Condition (iii) turns out to be exactly what is required in order that the variation $\delta x$ corresponding to $(\delta u, \delta y)$ in a minimal system also has compact support. Indeed the next result characterizes admissible variations. Since the transition matrix $\Phi^{\bar{u}}$ satisfies

$\Phi^{\vec{u}}(t, \sigma)=\Phi^{\bar{u}}(t, 0) \Phi^{\bar{u}}(0, \sigma)$,

we may write

$W_{v}(t, \sigma, \bar{u})=G(t, \bar{u}) H(\sigma, \bar{u})$

with $G(t, \vec{u})$ an $m \times k$ matrix and $H(\sigma, \bar{u})$ a $k \times m$ matrix (for a coordinate-free definition see [6]). 
Theorem 3.2. Let (1.1) be a minimal system. A variation $(\delta u, \delta y)$ of $(\bar{u}, \bar{y}) \in \Sigma_{\mathrm{e}}^{+}(0)\left(x_{0}\right)$ with supp $\delta u \subset(0, T)$ is admissible if and only if

$\int_{0}^{T} H(\sigma, \vec{u}) \delta u(\sigma) \mathrm{d} \sigma=0$.

Furthermore let $(\bar{u}, \bar{x}, \bar{y})$ be the corresponding element of $\Sigma_{\mathrm{i}}^{+}(0)\left(x_{0}\right)$. Then $(\delta u, \delta y)$ is admissible if and only if supp $\delta x \subset \operatorname{supp} \delta u$, and hence $\delta x(T)$ $=0$.

This result in turn demonstrates the existence of a great many admissible variations, since equation (3.3) represents only a finite number of constraints on the function $\delta u$.

The main technical result in [6] can now be expressed in the following way.

Theorem 3.3. Consider a minimal system (1.1). The system is Hamiltonian if and only if for any $(u, y)$ $\in \Sigma_{\mathrm{e}}^{+}(0)\left(x_{0}\right)$ and admissible variations $\left(\delta_{i} u, \delta_{i} y\right)$ of $(u, y)$ with compact support, $i=1,2$, we have

$\int_{0}^{\infty}\left[\delta_{2}^{\mathrm{T}} y(t) \delta_{1} u(t)-\delta_{1}^{\mathrm{T}} y(t) \delta_{2} u(t)\right] \mathrm{d} t=0$.

Remark. Note that (3.4) may be considered to be the dynamical generalization of the symmetry condition for a static input-output system $y_{j}=H_{j}(u)$, $j=1, \ldots, M$, to be a static reciprocal system of the form

$y_{j}=-\frac{\partial V}{\partial u_{j}}(u)$

with $V$ a potential. The 'only if' direction in Theorem 3.3 is easy. Consider a Hamiltonian system. Let $(u, x, y) \in \Sigma_{\mathrm{i}}^{+}(0)\left(x_{0}\right)$ and let $\left(\delta_{i} u, \delta_{i} x, \delta_{i} y\right), i=1,2$, be two variations. The following formula was proved in [10,11] (and can also be deduced from (2.4) and Theorem 2.3):

$$
\begin{aligned}
& \frac{\mathrm{d}}{\mathrm{d} t} \omega_{x(t)}\left(\delta_{1} x(t), \delta_{2} x(t)\right) \\
& \quad=\delta_{2}^{\mathrm{T}} y(t) \delta_{1} u(t)-\delta_{1}^{\mathrm{T}} y(t) \delta_{2} u(t) .
\end{aligned}
$$

Now let $\left(\delta_{i} u, \delta_{i} y\right)$ be admissible variations with compact support $\subset(0, T)$. Then it immediately follows from Theorem 3.2 that $\delta_{i} x(T)=\delta_{i} x(0)=$ $0, i=1,2$, and so (3.4) follows.

The 'if' direction is much harder. It is proved by substituting (2.5) into (3.4) and observing the constraints imposed by the fact that the variations are admissible and so satisfy equation (3.3). The result is the following lemma.

Lemma 3.4. Consider a minimal system (1.1) satisfying (3.4). For any $\tau>0$ there exists a piecewise constant control $\bar{u}$ on $[0, \tau)$ such that for any control $u$ with $u(t)=\bar{u}(t), t \in[0, \tau)$, we have

$W_{v}(t, \sigma, u)+W_{v}^{\mathbf{T}}(\sigma, t, u)=0, \quad t, \sigma \geqslant \tau$.

Thus if the system (1.1) is initialized at $\Psi_{\tau, 0}^{\pi}\left(x_{0}\right)$ (with $\Psi^{\bar{u}}$ the flow of the time-varying vector field $g_{0}+\sum_{j=1}^{m} \bar{u}_{j} g_{j}$ ), then every variational system is self-adjoint and so the system is Hamiltonian.

So far we have only considered minimal systems. However the self-adjointness as well as the variational criterion are expressed solely in terms of the (variational) input-output behavior of the system. Also a nonminimal system with external behavior $\Sigma_{\mathrm{e}}^{+}(0)\left(x_{0}\right)$ has a minimal realization with this same external behavior (where minimal means observable and accessible, cf. [13]). We therefore deduce the following result.

Corollary 3.5. Assume (1.1) is a nonlinear system satisfying $g_{0}\left(x_{0}\right) \in L_{0}\left(x_{0}\right)$, then the following are equivalent:

(a) Any minimal realization of (1.1) is Hamiltonian.

(b) The variational systems along all piecewise constant controls $u$ are self-adjoint.

(c) For every trajectory $(u, y) \in \Sigma_{\mathrm{e}}^{+}(0)\left(x_{0}\right)$ and for any two admissible variations $\left(\delta_{i} u, \delta_{i} y\right), i=1$, 2 , of $(u, y)$ with compact support, condition (3.4) is satisfied.

The condition $g_{0}\left(x_{0}\right) \in L_{0}\left(x_{0}\right)$, or equivalently $L\left(x_{0}\right)=L_{0}\left(x_{0}\right)$ simply means that an accessible realization is also strongly accessible, which, as we noted before, is a necessary condition for a system to be Hamiltonian. Without this condition we would obtain time-varying Hamiltonian systems, as discussed in [7].

Note that for minimal Hamiltonian realizations the internal energy $H_{0}$ need not be globally definable. On the other hand there always exists a globally. Hamiltonian realization, which is however only quasi-minimal (strongly accessible and weakly observable).

In a sense, the framework of initialized systems (1.1) is not the most natural one for our purposes, 
as illustrated by the cumbersome form of Lemma 3.4. The variational criterion especially can be stated in a more natural way for non-initialized systems (with $x(0)$ arbitrary). In this case, the behavior $\Sigma_{\mathrm{i}}$ is simply the set of all time functions $(u(t), x(t), y(t)), t \in(-\infty, \infty)$, satisfying (1.1) (see also [14]). The external behavior $\Sigma_{\mathrm{e}}$ is obtained by omitting $x(t)$. So $(u, y) \in \Sigma_{\mathrm{e}}$ if there exists an $x$ such that $(u, x, y) \in \Sigma_{\mathbf{i}}$.

We may now adapt Definition 3.1 to this setting by considering admissible variations with compact support on arbitrary intervals of $R$, and obtain a direct analogue of Theorems 3.2 and 3.3. We state the corresponding version of Theorem 3.3.

Theorem 3.6. Consider a minimal non-initialized system (1.1). The system is Hamiltonian if and only if for any $(u, y) \in \Sigma_{\mathrm{e}}$ all admissible variations $\left(\delta_{i} u, \delta_{i} y\right)$ of $(u, y)$ with compact support, $i=1,2$, satisfy

$\int_{-\infty}^{\infty}\left[\delta_{2}^{\mathrm{T}} y(t) \delta_{1} u(t)-\delta_{1}^{\mathrm{T}} y(t) \delta_{2} u(t)\right] \mathrm{d} t=0$.

Furthermore we have:

Theorem 3.7. Consider a minimal non-initialized Hamiltonian system $\Sigma$. Let $(u, y) \in \Sigma_{\mathrm{e}}$ and suppose that $(u, y)$ also belongs to the external behavior $\bar{\Sigma}_{\mathrm{e}}$ of some other minimal (not necessarily Hamiltonian) system, with the same state space $M$. Let $(D u, D y)$ be an admissible variation of $(u, y)$ of compact support where $(u, y)$ is viewed as an element of $\bar{\Sigma}_{\mathrm{e}}$. If every admissible variation $(\delta u, \delta y)$ of $(u, y)$ with compact support, where $(u, y)$ is viewed as element of $\Sigma_{e}$, satisfies

$\int_{-\infty}^{\infty}\left[D y^{\mathrm{T}}(t) \delta u(t)-\delta^{\mathrm{T}} y(t) D u(t)\right] \mathrm{d} t=0$,

then $(D u, D y)$ is also an admissible variation with compact support of $(u, y)$ viewed as element of $\Sigma_{e}$.

Theorems 3.6 and 3.7 have the following formal. interpretation. Consider the 'manifold' of maps $N_{M, m}$, defined as the union of all behaviour sets $\Sigma_{\mathrm{e}}$ as $\Sigma$ ranges over all non-initialized minimal systems (1.1) with state space $M$ and input space $R^{m}$. On this manifold we suppose the 'tangent space' to it at $(u, y)$, denoted $T_{(u, y)} N_{M, n}$, consists of all admissible variations $(\delta u, \delta y)$ of $(u, y)$ of compact support. Define the 'symplectic form' $\mu$ on $N_{M, m}$ as suggested by (3.7), i.e.,

$$
\begin{aligned}
& \mu_{(u, y)}\left(\left(\delta_{1} u, \delta_{1} y\right),\left(\delta_{2} u, \delta_{2} y\right)\right) \\
& \quad=\int_{-\infty}^{\infty}\left[\delta_{2}^{\mathrm{T}} y(t) \delta_{1} u(t)-\delta_{1}^{\mathrm{T}} y(t) \delta_{2} u(t)\right] \mathrm{d} t .
\end{aligned}
$$

Consider now a Hamiltonian system $\Sigma$ on $M$ with $m$ inputs. Then $\Sigma_{\mathrm{e}}$ is a 'submanifold' of $N_{M, m}$. Now Theorem 3.6 implies that the symplectic form $\mu$ is zero restricted to $\Sigma_{e}$, or equivalently, $\Sigma_{e}$ is an isotropic submanifold of $N_{M, n}$. On the other hand Theorem 3.7 implies that $\Sigma_{\mathrm{e}}$ is also a coisotropic submanifold. Hence the following corollary, stated as a conjecture in [11], is formally proven.

Theorem 3.8. A minimal non-initialized system $\Sigma$ on $M$ is Hamiltonian if an only if $\Sigma_{\mathrm{e}}$ is a Lagrangian submanifold of $N_{M, m}$ :

Finally we remark that the theory in this paper can be fully generalized to nonlinear systems where the control does not appear in an affine way. See [6] for details.

\section{References}

[1] R.W. Brockett, Finite Dimensional Linear Systems (Wiley, New York, 1970).

[2] R.W. Brockett, Control theory and analytical mechanics, in: C. Martin and R. Hermann, Eds., Geometric Control Theory, Vol. VII of Lie Groups; History, Frontiers and Applications (Math Sci Press, Brookline, MA, 1977).

[3] R.W. Brockett and A. Rahimi, Lie algebras and linear differential equations, in: L. Weiss, Ed., Ordinary Differential Equations (Academic Press, New York, 1972).

[4] P.E. Crouch, Hamiltonian realizations of finite Volterra series, Proc. MTNS 85, Stockholm (1985).

[5] P.E. Crouch and M. Irving, On finite Volterra series which admit Hamiltonian realizations, Math. Systems Theory 17 (1984) 293-318.

[6] P.E. Crouch and A.J. van der Schaft, Variational characterization of Hamiltonian systems, to appear.

[7] J.B. Goncalves, Realization theory of Hamiltonian systems, SIAM J. of Control 2A (1986).

[8] B. Jakubczyk, Hamiltonian realizations of nonlinear systems, Proc. MTNS 85, Stockholm (1985).

[9] R.M. Santilli, Foundations of Theoretical Mechanics I (Springer, New York, 1983).

[10] A.J. van der Schaft, Hamiltonian dynamics with external forces and observations, Math. Systems Theory 15 (1982) 145-168. 
[11] A.J. van der Schaft, System Theoretic Descriptions of Physical Systems, Doct. Diss. Univ. of Groningen (1983); appeared as CWI Tract No. 3 (CWI, Amsterdam, 1984).

[12] A.J. van der Schaft, Hamiltonian and quantum mechanical control systems, Memo. 546, Twente Univ. of Technology (Dec. 1985).
[13] H.J. Sussmann, Existence and uniqueness of minimal realizations of nonlinear systems, Math. Systems Theory 10 (1977) 263-284.

[14] J.C. Willems, System theoretic models for the analysis of physical systems, Richerche Automat. 10 (1979) 71-106. 